\title{
Single Nucleotide Polymorphisms (SNPs) of URAT1 (rs7932775) and ABCG2 (rs3825016) on Chronic Kidney Disease Patients with Hyperuricemia
}

\author{
Chunqing Li*, Qiong Tang, Hongwei Jiang, Jing Wu, Junlin Zhang, Fenglai Yuan, \\ Yuan Du, Haochang Du
}

Nephrology Department, The Third People’s Hospital of Wuxi, The Third Affiliated Hospital of Nantong University, Wuxi, China Email: *lichunqingwuxi@126.com

How to cite this paper: Li, C.Q., Tang, Q., Jiang, H.W., Wu, J., Zhang, J.L., Yuan, F.L., Du, Y. and Du, H.C. (2018) Single Nucleotide Polymorphisms (SNPs) of URAT1 (rs7932775) and ABCG2 (rs3825016) on Chronic Kidney Disease Patients with Hyperuricemia. Chinese Medicine, 9, 118-125. https://doi.org/10.4236/cm.2018.93007

Received: July 2, 2018

Accepted: July 30, 2018

Published: August 2, 2018

Copyright $\odot 2018$ by authors and Scientific Research Publishing Inc. This work is licensed under the Creative Commons Attribution International License (CC BY 4.0).

http://creativecommons.org/licenses/by/4.0/

\section{Abstract}

Background: More and more chronic kidney disease (CKD) patients are accompanied with hyperuricaemia. As is known, hyperuricaemia is an independent hazard of both cardiovascular diseases (CVD) and chronic kidney diseases. We aim at identifying Single Nucleotide Polymorphism (SNP) difference of hURAT1 (rs7932775) and ABCG2 (rs3825016) on CKD patient with hyperuricemia and/or gout. Methods: All forty-two CKD patients were divided into two groups: hyperuricemia, and control group. 24 hours urine sample and serum were prepared for testing biochemistry parameters. The polymerase chain reaction-restriction fragment length polymorphism (PCR-RFLP) method is used to analyze hURAT1 and ABCG2 single nucleotide polymorphisms in different groups. Results: 17 patients have CT SNP of hURAT1 (rs7932775) and 13 patients have CT SNP of ABCG2 (rs3825016) in hyperuricemia group, while only 5 persons and 6 persons have the same mutations in control group respectively. 7 patients have CT SNP of both hURAT1 (rs7932775) and ABCG2 (rs3825016) in hyperuricemia group, while only 2 persons have the same mutations in control group. CT mutation rates of hURAT1 (rs7932775) and ABCG2 (rs3825016) in hyperuricemia group were $60.7 \%(17 / 28)$ and 50\% (13/28) respectively, higher than that of control group $(35.7 \%(5 / 14)$ and $42.8 \%(6 / 14))$. What is more, Double SNP mutations in both hURAT1 (rs7932775) and ABCG2 (rs3825016) in hyperuricemia group were $25 \%$ (7/28), higher than that of control group $(14.2 \%, 2 / 14)$. Conclusion: There are higher mutation rates of CT SNP in hURAT1 (rs7932775) and/or ABCG2 (rs3825016) in hyperuricemia group. We can conclude that hyperuricemia is a high risk factor in progress of CKD, which is 
necessary to take measures of decreasing serum uric acid to delay CKD progress.

\section{Keywords}

Hyperuricemia, Chronic Kidney Disease (CKD), Single Nucleotide Polymorphisms (SNP), Human Urate Transport Protein (Hurat1), ATP Binding Transporter G Super Family (ABCG2)

\section{Introduction}

More and more chronic kidney disease (CKD) patients are accompanied with hyperuricaemia. As is known, hyperuricaemia is an independent hazard of both cardiovascular diseases (CVD) and chronic kidney diseases [1]. Hyperuricaemia and gout correlate with both these diseases [2] [3]. So those treatments which may lower serial uria and acute episode of gout can prevent and delay both cardiovascular diseases (CVD) and chronic kidney diseases [4]. Genetic variation of key enzymes of purine metabolism and urate transporter is closely related with occurrence and development of hyperuricaemia and gout [5] [6]. Genome-wide association studies (GWAS) have identified nearly 30 loci associated with urate concentrations that also influence the subsequent risk of gout. The ABCG2 Q141 $\mathrm{K}$ variant is highly likely to be causal and results in internalization of ABCG2 [7]. UP to now, there are at least three urate transporter genes, closely related with occurrence and development of hyperuricaemia: 1) ATP binding transporter G super family (ABCG2), 2) ATP binding transporter G super family, 3) human urate transport protein (HURAT1) [8]. The genetic contribution to the progression from hyperuricaemia to $\mathrm{CKD}$ remains relatively poorly understood.

But there have been not clearly understood about SNP identifying both URAT1 and CABG2 in hyperuricemia with CKD. So our aim is to identify and analyze Single Nucleotide Polymorphism (SNP) differences of hURAT1 (rs7932775) and ABCG2 (rs3825016) on CKD patient with hyperuricemia in order to identify uncommon genetic variants with increased penetrance that might provide opportunities for clinical translation.

\section{Methods}

1) All forty-two patients (M/F 24/22) were enrolled from outpatient and hospitalized from Jan. 2016 to Aug. 2017. Their mean age is 55 years. Patients were divided into two groups: Hyperuricemia, and control group. The groups were matched by age, body mass index, metabolic syndrome and use of anti-hypertensive medication.

2) Diagnostic criteria: serum creatine 123 - $701 \mathrm{umol} / \mathrm{L}$, eGFR $90-15 \mathrm{ml} / \mathrm{min}$, CKD 2 - 5 phase.

Chronic Kidney Disease (CKD) is defined by:

eGFR $<60 \mathrm{~mL} / \mathrm{min} / 1.73 \mathrm{~m}^{2}$ that is present for $\geq 3$ months or Markers of kid- 
ney damage present for $\geq 3$ months: a) Albuminuria $\geq 3 \mathrm{mg} / \mathrm{mmol}$; b) Urine sediment abnormalities; c) Structural or pathological abnormalities.

Hyperurecimia refers to the normal urine diet under the condition of two fasting uric acid levels. The male is higher than $420 \mathrm{umol} / \mathrm{L}$; the female is higher than $360 \mathrm{umol} / \mathrm{L}$.

Exclusion criteria: acute infection; liver function failure, myelosuppression, malignant tumor, children or elder persons ( $>80$ years).

3) Biochemistry parameters detection

24 hours urine sample and serum were prepared in both groups patients in for testing biochemistry parameters by Biochemical Analyzer.

4) Single Nucleotide Polymorphism (SNP) identifying of hURAT1 (rs7932775) and ABCG2 (rs3825016) [9].

Fasting blood samples were $(5 \mathrm{ml})$ extracted in the median cubital vein, DNA is extracted by method of salt bath, the polymerase chain reaction-restriction fragment length polymorphism (PCR-RFLP) method is used to analysis the case group and control group hURAT1, determination of ABCG2 single nucleotide polymorphisms. The identifying method is referred to the literature. The SNP identifying had been finished in Shanghai Genechem Co., Ltd.

Premier were designed as followed:

5) Statistical analysis

All data were statistically processed by SPSS 18.0 software, and the data were represented by $\mathrm{x} \pm \mathrm{s}$. Variance analysis was conducted. The comparison of the frequencies of genotypes and alleles, genotypes and alleles was conducted by using the direct count method.

\section{Results}

Biochemistry parameters in $24 \mathrm{~h}$ urinary and serum.

There have differences in $24 \mathrm{~h}$ urinary protein, creatine and UA between Control and Hyperuricemia group $(\mathrm{p}<0.05)$. But there have no difference in $24 \mathrm{~h}$ urinary UA between these groups ( $\mathrm{p}>0.05)$.

Single Nucleotide Polymorphism (SNP) of hURAT1 (rs7932775) and ABCG2 (rs3825016).

Table 1 shows Single Nucleotide Polymorphism (SNP) distribution of hURAT1 (rs7932775) and ABCG2 (rs3825016) indifferent groups patients. In Table 2, Table 3 and Figure 1, 17 patients have CT SNP of hURAT1 (rs7932775) in hyperuricemia group, while only 5 persons have the same mutations in control group. 13 patients have CT SNP of ABCG2 (rs3825016) in hyperuricemia group, while only 6 persons have the same mutations in control group; 7 patients have CT SNP of both hURAT1 (rs7932775)) and ABCG2 (rs3825016) in hyperuricemia group, while only 2 persons have the same mutations in control group.

From Figure 2, we can see, CT mutation rate of hURAT1 (rs7932775) in hyperuricemia group was $60.7 \%$ (17/28), which was higher than that of control group $(35.7 \%, 5 / 14)$. CT mutation rate of ABCG2 (rs3825016) in hyperuricemia 
Table 1. Single Nucleotide Polymorphism (SNP) of hURAT1 (rs7932775) and ABCG2 (rs3825016) in two groups.

\begin{tabular}{|c|c|c|c|c|c|}
\hline Patient & Gender & $\begin{array}{c}\text { Age } \\
\text { (year) }\end{array}$ & Underlying diseases & rs7932775 & rs3825016 \\
\hline 1 & M & 43 & l & $\mathrm{CC}$ & $\mathrm{CC}$ \\
\hline 2 & $\mathrm{~F}$ & 76 & $\mathrm{CHD}$, hypertension & $\mathrm{TT}$ & CT \\
\hline 3 & $\mathrm{~F}$ & 64 & MN hypertension & CT & $\mathrm{CC}$ \\
\hline 4 & $\mathrm{~F}$ & 33 & SLE & $\mathrm{CT}$ & $\mathrm{CC}$ \\
\hline 5 & $\mathrm{~F}$ & 33 & erythema nodosum & $\mathrm{CC}$ & $\mathrm{CC}$ \\
\hline 6 & $\mathrm{~F}$ & 65 & glomerulonephritis & TT & CT \\
\hline 7 & M & 43 & DKD T2DM hypertension & $\mathrm{TT}$ & $\mathrm{CC}$ \\
\hline 8 & $\mathrm{~F}$ & 60 & glomerulonephritis & CT & $\mathrm{CC}$ \\
\hline 9 & M & 62 & T2DM glomerulonephritis & $\mathrm{TT}$ & $\mathrm{CT}$ \\
\hline 10 & M & 62 & CAPD CKD5d T2-DM hypertension & $\mathrm{CC}$ & $\mathrm{CC}$ \\
\hline 11 & $\mathrm{~F}$ & 55 & Urinary tract infection biliary alculus & $\mathrm{CT}$ & $\mathrm{CT}$ \\
\hline 12 & $\mathrm{~F}$ & 71 & Pyelonephritis hypertension CHD & $\mathrm{TT}$ & CT \\
\hline 13 & $\mathrm{~F}$ & 58 & Pyelonephritis & $\mathrm{CC}$ & $\mathrm{CC}$ \\
\hline 14 & $\mathrm{~F}$ & 60 & l & CT & $\mathrm{CT}$ \\
\hline 15 & M & 77 & CKD Hypertension HUA & CT & $\mathrm{CC}$ \\
\hline 16 & $\mathrm{~F}$ & 62 & HUA T2DM Stroke & $\mathrm{CT}$ & $\mathrm{CT}$ \\
\hline 17 & $\mathrm{~F}$ & 44 & hypertension HUA IgAN & TT & CT \\
\hline 18 & M & 64 & CKD4 2-DM hypertension HUA & TT & CT \\
\hline 19 & M & 32 & $\begin{array}{c}\text { IgAN CKD3 } \\
\text { Postoperative of left kidney cancer Resection }\end{array}$ & CT & CT \\
\hline 20 & M & 54 & rhabdomyolysis interstitial nephritis & CT & $\mathrm{CC}$ \\
\hline 21 & M & 80 & HUA CDK5d hypertension T2DM CHD & CT & $\mathrm{CC}$ \\
\hline 22 & M & 67 & HUA & CT & CT \\
\hline 23 & M & 35 & HUA CAPD Hypertension C3 glomerulonephritis & CT & $\mathrm{CC}$ \\
\hline 24 & M & 70 & HUA gout & CT & $\mathrm{CC}$ \\
\hline 25 & $\mathrm{~F}$ & 56 & $\begin{array}{l}\text { HUA hypertension Postoperative of Heart valve } \\
\text { replacement }\end{array}$ & CT & CT \\
\hline 26 & M & 70 & HUA hypertension CKD3 phrase T2DM & TT & CT \\
\hline 27 & M & 37 & pyelonephritis & $\mathrm{CC}$ & $\mathrm{CC}$ \\
\hline 28 & M & 50 & Gout HUA & CT & CT \\
\hline 29 & M & 60 & HUA & TT & TT \\
\hline 30 & M & 43 & CKD5d phase hypotension & TT & $\mathrm{CC}$ \\
\hline 31 & M & 58 & MHD CKD5d phase & CT & CT \\
\hline 32 & M & 28 & Gout hypertension HUA & $\mathrm{CC}$ & $\mathrm{CC}$ \\
\hline 33 & $\mathrm{~F}$ & 47 & CKD4 phrase hypertension & $\mathrm{CT}$ & $\mathrm{CC}$ \\
\hline
\end{tabular}




\section{Continued}

\begin{tabular}{lllcll}
\hline 34 & $\mathrm{~F}$ & 40 & HUA HSPN Hypertension & TT & TT \\
35 & $\mathrm{~F}$ & 73 & CKD5 phrase T2DM Hypertension & CT & CC \\
36 & $\mathrm{~F}$ & 56 & HUA hypertension & TT & CT \\
37 & $\mathrm{M}$ & 22 & HUA Rhabdomyolysis & TT & CT \\
38 & $\mathrm{M}$ & 50 & Gout T2DM & CT & CT \\
39 & $\mathrm{M}$ & 70 & CKD4 Phrase gout Hypertension & CT & CC \\
40 & $\mathrm{M}$ & 26 & HUA urinary tract infection & CT & CC \\
41 & $\mathrm{M}$ & 37 & CAPD CKD5d phrase Hypertension & TT & CT \\
42 & $\mathrm{~F}$ & 62 & CKD4 phrase Gout Hypertension & CT & CC \\
\hline
\end{tabular}

Patient 1 - 14 refer to control group, patients 15 - 42 refer to hyperuricemia group patients. 17 patients have CT SNP of hURAT1 (rs7932775) and 13 patients have CT SNP of ABCG2 (rs3825016) in hyperuricemia group, while only 5 persons and 6 persons have the same mutations in control group respectively. 7 patients have CT SNP of both hURAT1 (rs7932775) and ABCG2 (rs3825016) in hyperuricemia group, while only 2 persons have the same mutations in control group.

Table 2. SNP for rs 7932775 and rs 3825016.

\begin{tabular}{ccc}
\hline Premier & Series $\left(5^{\prime}\right.$ to $\left.3^{\prime}\right)$ & Length \\
\hline EX031-E1-F & CAGGACAGGATACCCAGATGGAG & $261 \mathrm{bp}$ \\
EX031-E1-R & CAGCCTCACCTGAGCACAGTGG & \\
EX031-E3-F & GTCTCCATCATGTGGCTGTGTAC & $299 \mathrm{bp}$ \\
EX031-E3-R & CTGCGGTCATAGACCCAGCCATC & \\
& \\
SNP & message & \\
rs7932775 & AGAAATGGGGGCTCTGCGCTCAGCC $[C / G / T]$ TGGCCGTGCTGGGGCTGG \\
& GCGGGGT \\
rs3825016 & GTGGCTGGCGGAAGCGGCGGCACTG $[A / G]$ TGGGGCCTCTGGTTGGGGC \\
& CCGGCG
\end{tabular}

Table 3. Biochemistry parameters in urine and serum between groups.

\begin{tabular}{ccccc}
\hline & 24 h urinary protein & BUN & Creatine & UA \\
\hline Control & $1.09 \pm 1.58$ & $7.26 \pm 4.1$ & $137.22 \pm 148.14$ & $282.36 \pm 129.28$ \\
Hyperuricemia & $0.88 \pm 0.96$ & $11.83 \pm 8.16$ & $303.95 \pm 299.21$ & $503.18 \pm 119.61$ \\
$\mathrm{p}$ & 0.622 & 0.074 & 0.020 & $2283.64 \pm 1287.12$ \\
\hline
\end{tabular}

group was $50 \%(13 / 28)$, higher than that of control group $(42.8 \%, 6 / 14)$. What is more, Double SNP mutations in both hURAT1 (rs7932775) and ABCG2 (rs3825016) in hyperuricemia group was 25\% (7/28), higher than that of control group $(14.2 \%, 2 / 14)$.

\section{Discussion}

A number of epidemiological reports link hyperuricemia with multiple disorders, 


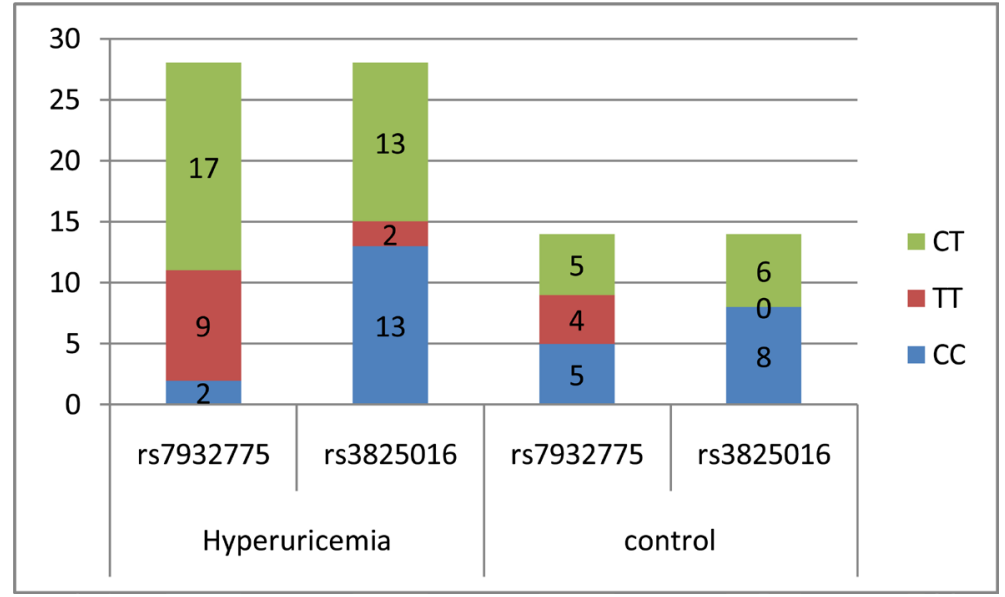

Figure 1. Single Nucleotide Polymorphism (SNP) of hURAT1 (rs7932775) and ABCG2 (rs3825016) in different groups.

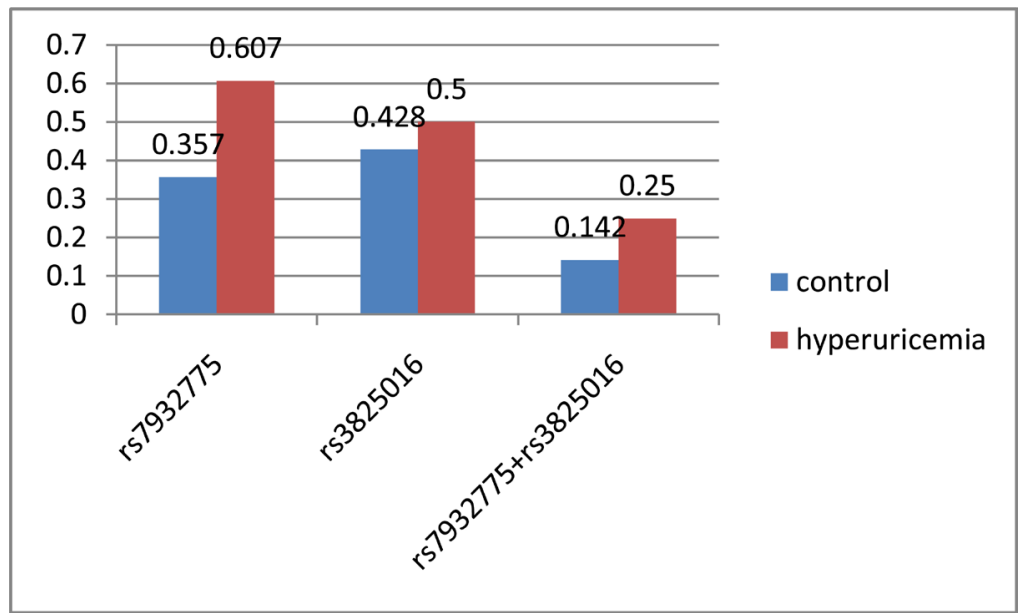

Figure 2. Rate of mutation of SNP of hURAT1 (rs7932775) and ABCG2 (rs3825016) in different groups.

such as kidney diseases, cardiovascular diseases and diabetes. Recent studies also showed that expression and functional changes of urate transporters are associated with hyperuricemia. Uric acid transporters are divided into two categories: urate reabsorption transporters, including urate anion transporter 1 (URAT1), organic anion transporter 4 (OAT4) and glucose transporter 9 (GLUT9), and urate excretion transporters, including OAT1, OAT3, urate transporter (UAT), multidrug resistance protein 4 (MRP4/ABCC4), ABCG-2 and sodium-dependent phosphate transport protein. In the kidney, uric acid transporters decrease the reabsorption of urate and increase its secretion. These transporters' dysfunction would lead to hyperuricemia. As the function of urate transporters is important to control the level of serum uric acid, studies on the functional role of uric acid transporter may provide a new strategy to treat hyperuricemia associated diseases, such as gout, chronic kidney disease, hyperlipidemia, hypertension, coronary heart disease, diabetes and other disorders. Tan [10] suggested that URAT1 inhibitors bind to a common site in the core of the transporter and sterically 
hinder the transit of uric acid through the substrate channel, albeit with vastly different potencies and with differential interactions with specific URAT1 amino acids.

We aimed to identify and explore Single Nucleotide Polymorphism (SNP) mutations of hURAT1 (rs7932775) and ABCG2 (rs3825016) on CKD patients to provide a new strategy to treat hyperuricemia associated CKD. Genome-wide association studies (GWAS) have confirmed the importance of urate excretion in the control of serum urate levels and the risk of gout and have identified the kidneys, the gut and the liver as sites of urate regulation. Human urate transporter 1 (URAT1) is a member of the organic anion transporter family (SLC22A12) that primarily regulates the renal tubular reabsorption of uric acid. Cho [11] found five newly described SNPs (rs7929627, rs75786299, rs3825017, rs11602903 and rs121907892) in the hURAT1 gene are significantly associated with uric acid level. Sun [12] explored the effect of urate transporter 1 (URAT1) polymorphisms on the hypertensive patients with hyperuricemia and the uricosuric action of losartan therapy among hypertensive patients with hyperuricemia, suggesting that URAT1 rs3825016 and rs1529909 polymorphisms influence the uricosuric action of losartan. Chen et al. [13] in GWAS results showed 36 SNPs to be strongly associated with gout compared to controls (all p-values \& lt; 10 $(-7)$ ), and ABCG2 gene contributed to hyperuricemia but also gout, and that it was involved in the inflammation dysregulation via augmented IL-8 release in EC.

Up to now, we have known little about SNP identifying both URAT1 and CABG2 in hyperuricemia with CKD. Our work shows that there are higher mutation rate of SNPs of hURAT1 (rs7932775) or ABCG2 (rs3825016) in CKD with hyperuricemia patients compared to controls. We can conclude that hURAT1 (rs7932775) or ABCG2 (rs3825016) may play a part in progress of CKD by triggering oxidative stress, which is necessary to take decrease serum uric acid to delay CKD progress. We also found the higher SNP mutation of both hURAT1 (rs7932775) and ABCG2 (rs3825016), suggesting the common mechanisms in triggering CKD progress. In the following work, we plan to test SNP of hURAT1 (rs7932775) or ABCG2 (rs3825016) in CKD patients by serum uric acid-lowering drugs Febuxostat and Benzbromarone respectively to explore the common mechanisms from hyperuricemia to CKD progress. SNP mutations patients on followed-up study are more susceptible to cause gout flare and CKD than no-mutation patients, to whom we pay close attention and take intervening measures by giving decreasing serum urice acid medicine. It is worth of further research to explore the exact mechanisms of SNP of URAT1 (rs7932775) and ABCG2 (rs3825016) causing gout and CKD progress.

\section{Funding}

The work was funded by the project of twelfth group of six talent peak project of Jiangsu Province (No-2015-WSN-089). 


\section{Conflicts of Interest}

The authors declare no conflicts of interest regarding the publication of this paper.

\section{References}

[1] Srivastava, A., Kaze, A.D., Mcmullan, C.J., et al. (2018) Uric Acid and the Risks of Kidney Failure and Death in Individuals with CKD. American Journal of Kidney Diseases, 71, 362-370. https://doi.org/10.1053/j.ajkd.2017.08.017

[2] Li, C.C., Chien, T.M., Wu, W.J., et al. (2018) Uric Acid Stones Increase the Risk of Chronic Kidney Disease. Urolithiasis, 1-5. https://doi.org/10.1007/s00240-018-1050-1

[3] Garofalo, C., De Stefano, T., Vita, C., et al. (2018) Hyperuricaemia and Chronic Kidney Disease. Giornale italiano di nefrologia, 35, No. 1.

[4] Johnson, R.J., Bakris, G.L., Borghi, C., et al. (2018) Hyperuricemia, Acute and Chronic Kidney Disease, Hypertension, and Cardiovascular Disease: Report of a Scientific Workshop Organized by the National Kidney Foundation. American Journal of Kidney Diseases, 71, 851-865. https://doi.org/10.1053/j.ajkd.2017.12.009

[5] Dalbeth, N., Stamp, L.K. and Merriman, T.R. (2017) The Genetics of Gout: Towards Personalised Medicine? BMC Medicine, 15, 108. https://doi.org/10.1186/s12916-017-0878-5

[6] Major, T.J., Dalbeth, N., Stahl, E.A., et al. (2018) An Update on the Genetics of Hyperuricaemia and Gout. Nature Reviews Rheumatology, 14, 341-353. https://doi.org/10.1038/s41584-018-0004-x

[7] Merriman, T. (2017) Genomic Influences on Hyperuricemia and Gout. Rheumatic Disease Clinics of North America, 43, 389-399. https://doi.org/10.1016/j.rdc.2017.04.004

[8] Xu, L., Shi, Y., Zhuang, S., et al. (2017) Recent Advances on Uric Acid Transporters. Oncotarget, 8, 100852-100862. https://doi.org/10.18632/oncotarget.20135

[9] Jiri, M., Zhang, L., Lan, B., et al. (2016) Genetic Variation in the ABCG2 Gene Is Associated with Gout Risk in the Chinese Han Population. Clinical Rheumatology, 35, 159-163. https://doi.org/10.1007/s10067-015-3105-9

[10] Tan, P.K., Liu, S., Gunic, E., et al. (2017) Discovery and Characterization of Verinurad, a Potent and Specific Inhibitor of URAT1 for the Treatment of Hyperuricemia and Gout. Scientific Reports, 7, 665. https://doi.org/10.1038/s41598-017-00706-7

[11] Cho, S.K., Kim, S., Chung, J.Y., et al. (2015) Discovery of URAT1 SNPs and Association between Serum Uric Acid Levels and URAT1. BMJ Open, 5, e9360. https://doi.org/10.1136/bmjopen-2015-009360

[12] Sun, H., Qu, Q., Qu, J., et al. (2015) URAT1 Gene Polymorphisms Influence Uricosuric Action of Losartan in Hypertensive Patients with Hyperuricemia. Pharmacogenomics, 16, 855-863. https://doi.org/10.2217/pgs.15.52

[13] Chen, C.J., Tseng, C.C., Yen, J.H., et al. (2018) ABCG2 Contributes to the Development of Gout and Hyperuricemia in a Genome-Wide Association Study. Scientific Reports, 8, 3137. https://doi.org/10.1038/s41598-018-21425-7 